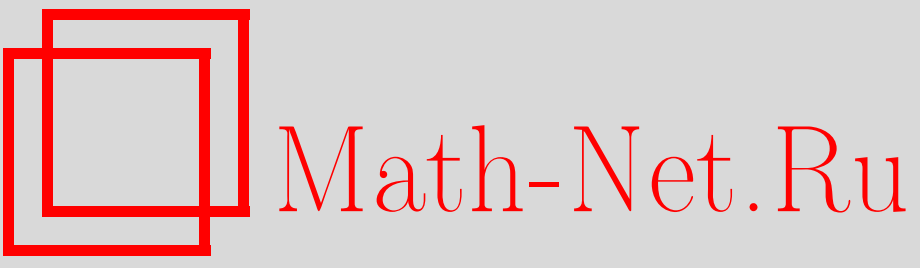

А. Г. Витушкин, 13-я проблема Гильберта и смежные вопросы, УМH, 2004, том 59, выпуск 1, 11-24

DOI: https://doi.org/10.4213/rm698

Использование Общероссийского математического портала Math-Net.Ru подразумевает, что вы прочитали и согласны с пользовательским соглашением

http://www.mathnet.ru/rus/agreement

Параметры загрузки:

IP : 54.196 .121 .252

26 апреля 2023 г., 14:50:41 


\title{
13-Я ПРОБЛЕМА ГИЛЬБЕРТА И СМЕЖНЫЕ ВОПРОСЫ
}

\author{
А. Г. Витушкин
}

\begin{abstract}
Обсуждаемая проблема Гильберта посвящена изучению решений алгебраических уравнений. Задача состоит в том, чтобы получить оценку сложности алгебраической функции. Проблема остается открытой. Имеется лишь несколько частных алгебраических результатов, и в то же время проблема стимулировала ряд исследований по теории функций с их последующими приложениями. Наиболее ярким результатом этого цикла является теорема Колмогорова о суперпозициях непрерывных функций.

Библиографиия: 87 названий.
\end{abstract}

\section{СОДЕРЖАНИЕ}

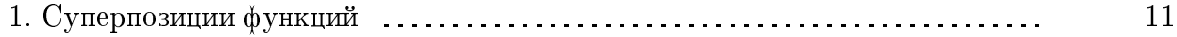

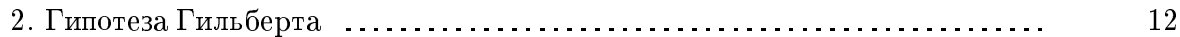

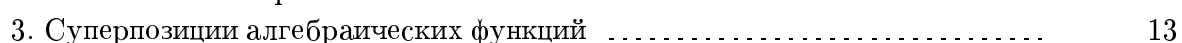

4. Суперпозиции аналитических функций .......................... 13

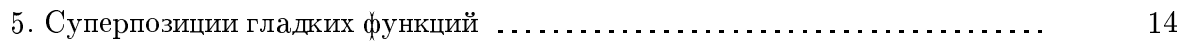

6. Суперпозиции непрерьвных функций . . . . . . . . . . . . . . . . . . 15

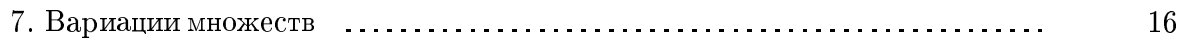

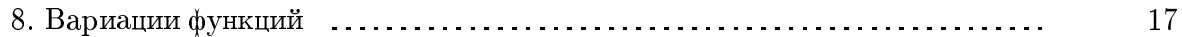

9. Оценки $\varepsilon$-энтропии компакта гладких функций … . . . . . . . . . . . . . . . . 17

10. Пример оценки сложности алгоритмов ............................ 18

11. Кодирование сигналов с конечным спектром ...................... 19

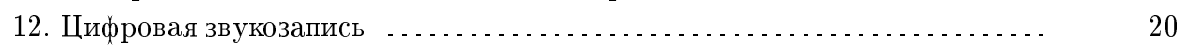

Список литературы .................................................... 21

1. Суперпозиции функций. Постановки задач и результаты будут формулироваться в терминах суперпозиций функций, и потому напомним соответствующее определение. Функция $f\left(x_{1}, \ldots, x_{n}\right)$ является суперпозицией функций $\left\{f_{\alpha_{i}}\left(y_{\alpha_{i}, 1}, \ldots\right.\right.$, $\left.\left.y_{\alpha_{i}, k}\right)\right\}\left(i=0,1, \ldots, s ;\right.$ мультииндекс $\alpha_{i}=\left(\beta_{0}, \ldots, \beta_{i}\right) ; \beta_{0}=0 ; \beta_{j}=1, \ldots, k$ при $j>0$; каждый из аргументов $y_{\alpha_{s}, j}$ равен одной из координат $\left.x_{1}, \ldots, x_{n}\right)$, если заменяя последовательно аргументы $y_{\alpha_{i}}$ функциями $f_{\alpha_{i}}\left(y_{\alpha_{i}, 1}, \ldots, y_{\alpha_{i}, k}\right)$, мы получим заданную функщию $f\left(x_{1}, \ldots, x_{n}\right)$.

Приведем один пример использования суперпозиций функций в вычислительной математике. Пусть задан алгоритм вычисления некоторой функции от $n$ переменных и 
требуется указать другой алгоритм, позволяющий вычислять эту функцию одновременно с ее градиентом. Такая задача возникает, например, при вычислении экстремума функции. Спрашивается, сколь сильно возрастет число вьполняемых арифметических операций. Обычно отвечают, что число операций возрастет, по крайней мере, в $n$ раз. Это неверно.

К. В. Ким заметил, что имеется возможность решить эту задачу, увеличивая число операций лишш в 3-4 раза (имеется в виду асимптотика при больших $n$ ). Идея состоит в следуюшем. Всякая формула для вьполнения последовательности арифметических операций - это суперпозиция функщий от двух переменных. Поэтому формула для вычисления каждой из частных производных исходной функции будет суперпозицией тех же представляюших функций от двух переменных и их частных производных. Сложность выгисления функции двух переменных вместе с ее градиентом может превосходить приблизительно не более чем в три раза сложность вычисления функции без градиента. Вычисление значений представляющих функций и их градиентов это самая большая часть работы. Поэтому в целом число операций возрастает не в $n$ раз, а сушественно меньше. Ким реализовал эту идею компьютерной программой (1984 г., [1]).

2. Гипотеза Гильберта. Решение алгебраического уравнения степени $n \leqslant 4$ задается формулой, содержашей лишь радикалы и арифметические операции, и потому является суперпозицией функций двух переменных. При $n>4$ алгебраическая подстановка, назьваемая преобразованием Чирнгаузена, задаваемая формулой, содержашей лиш радикалы и арифметические операции, приводит общее алгебраическое уравнение $n$-й степени $x^{n}+a_{1} x^{n-1}+\cdots+a_{n}=0$ к виду $y^{n}+b_{n-4} y^{n-4}+\cdots+$ $b_{n-1} y+1=0$. Здесь $\left\{a_{i}\right\}$ и $\left\{b_{i}\right\}-$ комплексные числа. Получается, что решение алгебраического уравнения $n$-й степени при $n<7$ является суперпозицией функций двух переменных, а при $n \geqslant 7$ - суперпозицией функций $(n-4)$ переменных.

В частности, решение полного алгебраического уравнения 7-й степени является суперпозицией арифметических операций, радикалов и одной алгебраической функции трех переменных, являюшейся решением уравнения $f^{7}+x f^{3}+y f^{2}+z f+1=0$. Дальнейшее упрошение этого уравнения посредством алгебраических подстановок, по-видимому, невозможно.

В знаменитом списке проблем Гильберта (1900 г., [2]) под номером 13 эта гипотеза формулируется так: решение общего уравнения 7-й степени не представимо суперпозицией непрерывных функиий двух переменных. Здесь и далее, говоря о решении алгебраического уравнения, мы будем иметь в виду какую-либо однозначную ветвь решения. Однако, судя по комментариям Гильберта к этой проблеме, нет уверенности в том, что он имел в виду то же самое.

Проблема не менее привлекательна, если в формулировке гипотезы иметь в виду полное (семизначное) решение уравнения и многозначные непрерьвные функции двух комплексных переменных. Такое понимание гипотезы выделяет топологический аспект проблемы. Возможно, что “сложность" дискриминантной кривой полного решения может быть препятствием для представления решения суперпозицией функций только двух переменных.

И вообще, эту проблему понимали по-разному. Гильберт в своих комментариях [2] связьвал эту проблему с потребностями номографии (номограмма - это набор плоских кривых, предназначенный для графического вычисления значений какой-либо простейшей функции нескольких переменных). Позже он занимался суперпозищиями 
алгебраических функций [3]. Виман [4] и Чеботарёв [5] рассчитьвали на возможное развитие теории Галуа. Колмогоров в молодости относил эту проблему к теории аналитических функций и советовал П.С. Александрову заняться этой задачей, акцентируя внимание на топологическом аспекте проблемы (1932 г., [6; с. 444]).

Колмогоров доказал, что всякая непрерывная функиия нескольких переменньх может быть представлена суперпозичией непрерывных функиий одной переменной и операчии сложсения (1957 г., [7]). Получается так, что функций нескольких переменных как бы вовсе и нет. Имеются лишь несложные комбинации функций одной переменной. K сожалению, представляющие функции заведомо плохи, они всего лишь непрерывны и не могут быть выбраны дифференцируемьми даже в тех случаях, когда речь идет о представлении аналитической функции (1964 г., [8]).

Если серьезно, теорема Колмогорова - это блестящий образеч мастерства. В частности, теорема показьвает, что гипотеза Гильберта ошибочна.

Однако необходимо отметить, что алгебраическое ядро проблемы осталось незатронутым. Можно рассчитьвать на положительное решение проблемы, т.е. возможно, что решение уравнения 7-й степени не представимо суперпозицией функиий двух переменных (разумеется при условии какой-либо гладкости этих функций). Неизвестно, существует ли хотя бы какая-нибудь алгебраическая функция $n$ переменных $(n>2)$, не представимая суперпозицией алгебраических функций меньшего числа переменных. Не исключено также, что всякая алгебраическая функция нескольких переменных является суперпозицией алгебраических функций одной переменной и арифметических операций. Таким образом, проблема остается открытой и диапазон вопросов по большому счету столь жсе широк, как и в начале XX века.

3. Суперпозиции алгебраических функций. Сам Гильберт занимался алгебраическим аспектом проблемы. Он показал, что решение обшего алгебраического уравнения 9-й степени представимо суперпозицией алгебраических функций 4 переменных (1927 г., [3]). (Напомним, что преобразование Чирнгаузена дает представление решения уравнения 9-й степени суперпозицией функций пяти переменных.)

Имеется ряд результатов о решении уравнений более высоких степеней [4], [5], [9], [10], аналогичных этой теореме Гильберта.

Приведем один результат другого рода, связанньй с поиском подходов к положительному решению проблемы. А. Г. Хованский показал (1971 г., [11], [12]), что решение уравнения $f^{5}+x f^{2}+y f+1=0$ не может быть представлено суперпозицией целых алгебраических функций одной переменной и полиномов от произвольного числа переменных. Напомним, что преобразование Чирнгаузена сводит обшее алгебраическое уравнение 5-й степени к уравнению с одним параметром, т.е. функция Хованского является суперпозищией алгебраических функций одной переменной и арифметических операций. Этот пример показьвает, что запрет использовать операцию деления является сушественным.

4. Суперпозиции аналитических функций. Никто до Колмогорова не посмел усомниться в справедливости гипотезы Гильберта. Поэтому суперпозищиями непрерьвных функций не занимались. (Исключением была лишь ошибочная работа Л. Бибербаха (1931 г., [13]).) Рассчитьвая на положительное решение проблемы, естественно для начала ограничить класс представляюших функций, рассматривая, например, суперпозиции достаточно гладких функций.

Легко показать, что сушествует аналитическая функция трех переменных, не представимая суперпозицией аналитических функций двух переменных. Идея доказатель- 
ства в том, что число частных производных порядка $p$ функции трех переменных пропорционально $p^{2}$, а число частных производных того же порядка $p$ функции двух переменных пропорционально $p$, и потому функций трех переменных "больше", чем суперпозиций функций двух переменных.

Имеются и конкретные примеры. Так, А. Островский (1920 г., [14]) показал, что аналитическая функция $\xi(x, y)=\sum_{n=1}^{\infty} \frac{x^{n}}{n^{y}}$ не может быть представлена суперпозицией бесконечно дифференцируемых функций одной переменной и алгебраических функций любого числа переменных. Доказательство этого утверждения основано на том, что функция $\xi(x, y)$ не удовлетворяет никакому алгебраическому уравнению в частных производных, т.е. уравнению вида $\Phi\left(\xi, \frac{\partial \xi}{\partial x}, \frac{\partial \xi}{\partial y}, \ldots, \frac{\partial^{\mu+\lambda} \xi(x, y)}{\partial x^{\mu} \partial y^{\lambda}}\right)=0$, где $\Phi$ - это полином с постоянньми коэффициентами от функции $\xi$ и всех ее частных производных до некоторого конечного порядка. С другой стороны, относительно просто доказывается, что всякая функция от двух переменных, являющаяся суперпозицией указанного вида, удовлетворяет такому дифференциальному уравнению.

5. Суперпозиции гладких функций. В 50-е - 60-е годы в связи с обсуждаемой проблемой Гильберта появился большой цикл работ по теории функций с последующими разнообразными приложениями. Первьй результат этого цикла был получен в семинаре А.С. Кронрода (об этом семинаре см. [15]). Было доказано, что существует непрерывно дифференцируемая функция трех переменных, не представимая суперпозицией непрерывно дифференцируемых функций двух переменных (1954 г., [16]).

Доклад по этой работе в семинаре А.С. Кронрода запомнился мне особо. Тогда (1953 г.) мехмат располагался в старом здании университета на углу улиц Герцена и Моховой. Был тепльй весенний день, и Кронрод предложил провести семинар на свежем воздухе, в университетском дворе. В таких случаях семинар проходил в небольшом палисаднике около памятника Ломоносову. Сидели мы на ограждающем памятник парапете, в доске необходимости не было, нас было мало, и мы легко понимали друг друга и так. Занятия, как правило, проходили шумно, и иногда это собирало зрителей.

Зрители развлекались также необычным видом памятника. Ломоносов был изображен в полньй рост со свитком в левой руке, кисть, державшая свиток, прижата к верхней части бедра. При взгляде на статую в профиль справа о торчащем свитке приходилось лишш догадьваться, что это такое. Первокурсницы, те, которые понаблюдательнее, взглянув на памятник, краснели и смущенно отворачивались. Со временем свиток убрали. Теперь памятник основателю Московского университета вьглядит пристойно.

Эта работа по суперпозищиям дала мне повод познакомиться с Колмогоровым и стать его аспирантом. Я попросил его представить пару моих заметок [16], [17] в ДАН. Выслушав формулировку результата, он, произнеся обычное свое длинное “э ...", добавил: “Да-да, это верно, и я понимаю, как это можно сделать”. Я был несколько обескуражен. Присутствовавший при разговоре Павел Сергеевич Александров успокоил меня: “Толя, не обижайтесь. Андрей Николаевич понимает все”.

Через неделю после этого разговора, когда я пришел к Колмогорову за ответом, меня ожидал сюрприз другого рода. Он полностью переписал обе заметки и напечатал их на машинке, объяснив таким своеобразным способом, как следует излагать материал и оформлять работу для публикации. 
Впоследствии, когда возникла необходимость написания обзоров и появилось желание по возможности усилить имеюшиеся результаты, из этой теоремы получилось следующее утверждение: если в качестве меры сложности функции принять отношение числа ее аргументов к порядку ее гладкости, то получится, что почти всякая функиия заданной сложности (за исключением функиий, составляющих множество 1-й категории) не может быть представлена суперпозицией функций меньшей сложсности [18].

6. Суперпозиции непрерывных функций. Колмогоров говорил, что 13-я проблема Гильберта - это хороший материал для студентов. Со временем стало ясно, что эта задача не только для студентов, и он сам увлекся ею. Решающим результатом была работа Колмогорова о возможсности представления непрерьвной функции нескольких переменных суперпозицией функций от трех переменных $(1956$ г., [19]).

Колмогоров рассказьвал, что идея конструкции появилась у него, когда он, по привычке просматривать иногда старые журналы, обратил внимание на статью А.С. Кронрода [20], в которой среди прочего рассматривались функциональные деревья. Дерево функции - это пространство компонент ее уровней. Дерево одномерно и ациклично и потому гомеоморфно укладывается на плоскость. Значения функции естественньм образом переносятся на ее дерево, и тем самьм функция многих переменных оказьвается в определенном смысле функцией только двух переменных. При построении суперпозищий потребовалось введение еше одной переменной, тем самым образовались суперпозиции функций трех переменных.

Этот результат Колмогорова был сенсацией: идея Гильберта охарактеризовать сложность решения алгебраического уравнения в терминах суперпозиций непрерывныл $x$ функций оказалась несостоятельной. По существу стало понятно, что его гипотеза об уравнении 7-й степени тоже ошибочна. Однако опровержение гипотезы потребовало дополнительных усилий.

Колмогоров был превосходньм научным руководителем. Работая со студентами, он умел предоставить ученику возможность, как говорится, сыграть соло. Так было и в данном случае. Студент 3-го курса Дима Арнольд, усовершенствовав колмогоровскую конструкцию, доказал, что всякую непрерьвную функцию нескольких переменных, в частности, и решение полного алгебраического уравнения 7-й степени, можно представить в виде суперпозиции непрерывных функций от двух переменных, показав тем самьм ошибочность гипотезы Гильберта (1957 г., [21], 1958 г., [22], 1959 г., [23]).

Колмогоров не был бы Колмогоровым, если бы оставил эту работу незавершенной. Вскоре, следуя правилу доводить результат до блеска, он, отказавшись от использования функциональных деревьев, нашел новую и, по-видимому, простейшую конструкцию и доказал следуюшее: всякая функция $f$, определенная и непрерьвная на $n$-мерном кубе, представима суперпозицией вида

$$
f\left(x_{1}, \ldots, x_{n}\right)=\sum_{i=1}^{2 n+1} f_{i}\left(\sum_{j=1}^{n} \phi_{i, j}\left(x_{j}\right)\right)
$$

где все функщии $\left\{f_{i}\right\}$ и $\left\{\phi_{i, j}\right\}$ непрерывны на $\mathbb{R}^{1}$ и при этом функции $\left\{\phi_{i, j}\right\}$ не зависят от выбора функции $f$ (1957 г., [7]). 
Имеется много комментариев, уточнений этой теоремы и иллюстраций ее доказательства [8], [15], [18], [24]-[40]. Нашлось лишш одно усовершенствование колмогоровской конструкции. Б. Л. Фридман (1967 г., [34]) доказал, что функции $\left\{\phi_{i, j}\right\}$ могут быть выбраны удовлетворяюшими условию Липшица с показателем 1 . Однако следует отметить, что если в колмогоровской формуле заменить функции $\left\{\phi_{i, j}\right\}$ какими-либо непрерьвно дифференцируемыми функциями, то окажется, что даже не всякая аналитическая функция может быть представлена этой формулой (1964 г., [8]).

Теорема Колмогорова завершает цикл работ по суперпозициям непрерывных функций. Разумеется, в теории функций возникают новые вопросы по данной теме. Например, всякая ли аналитическая функция двух переменных мохст быть представлена суперпозицией непрерывно дифференцируемых функций одной переменной и операчии сложения.

Предвестником обсуждаемых здесь результатов о суперпозициях и гладких и непрерьвных функций могла бы стать работа А.А. Милютина (1951 г.). Он доказал, что пространство функций $n$ переменных при всяком $n$ изоморфно пространству функций одной переменной, подчеркнув,что изоморфизм невозможен, если дополнительно требовать, что гладкие функции переходят в гладкие функции (вторая часть теоремы получалась сравнением мошностей $\varepsilon$-сетей соответствуюших компактов гладких функций). Могла бы стать предвестником, но не стала. А. Пельчинский сказал, что эта работа ошибочна. Ее не стали печатать, и о ней забыли. Через 15 лет Г. М. Хенкин получил такой же результат. Узнав о работе А. А. Милютина (наверное, опять же от А. Пельчинского), он, любопытства ради, отыскал в библиотеке мехмата рукопись Милютина. Оказалось, что никаких ошибок там нет, и Хенкин организовал публикацию работы Милютина (1966 г., [41]).

7. Вариации множеств. Обсудим теперь некоторые идеи, возникшие в связи с задачей о суперпозициях гладких функщий. Напомним [42]-[44], что подмножеству $n$-мерного пространства сопоставляются вариации порядков от 0 до $n$. $k$-я вариацияэто интеграл по пространству всех плоскостей размерности $n-k$ от числа компонент пересечения плоскости с данньм множеством.

Рассмотрим пример множества в $\mathbb{R}^{3}$, которое вьплядит, например, как обычная веревка. Для него определены четыре вариации. Нулевая вариация равна числу кусков веревки. Первая вариация равна длине веревки в обычном житейском смысле этого слова (хаусдорфова длина веревки равна бесконечности). Вторая вариация веревки равна площади ее поверхности. А последняя, третья вариация - это объем данного множества.

Колмогоров обратил внимание на то, что старшая отличная от 0 вариация множества равна его мере Фавара того же порядка (мера Фавара определяется так же, как и вариация, с единственньм отличием - интегрировать нужно не число компонент, а число точек). Доказьвать здесь нечего, это всего лишш наблюдение. Однако все равно приятно - для всякого множества одна из вариаций численно равна мере соответствующего порядка.

Вариации хороши тем, что они характеризуют “протяженность" множества одновременно во всех размерностях. Это обстоятельство может быть использовано при оценке сложности тех или иных математических объектов. В частности, это свойство вариаций было удачно использовано для описания массивности различных компактов гладких функций. Поясним, что вариации множества функций определяются как вариации подходяшим образом подобранной проекции данного множества функций в $\mathbb{R}^{n}$, 
например, проекции, полученной ограничением функций на достаточно плотную сеть точек.

Оценку вариаций множества можно получить, приближая его алгебраической поверхностью и используя имеющиеся оценки числа компонент алгебраических поверхностей. Имеются работы И.Г. Петровского и О.А. Олейник, содержашие точную оценку чисел Бетти (в частности, и оценку числа компонент) вещественной алгебраической гиперповерхности ([45], [46], 1952 г.). Именно эти точные оценки и техника, обретенная в работе с вариациями множеств, составили основу доказательства приведенной выше теоремы о суперпозициях гладких функций (см. раздел 5).

8. Вариации функций. Вариации множеств появились как обобщение понятия кронродовских вариаций функций двух переменных [20]. При этом в терминах вариаций множеств получилось удачное определение вариаций функций произвольного числа переменных. Для функции $n$ переменных определяются вариации всех порядков от 1 до $n$. $k$-я вариация функии $f$-это интеграл по всем действительньм значениям $t$ от $(k-1)$-й вариации множества уровня $f=t$. Почти все результаты А.С. Кронрода по вариациям функций двух переменных [20] без труда переносятся на функции произвольного числа переменных. Единственным исключением является доказательство того, что линейная вариация $n$ раз непрерывно дифференцируемой функции $n$ переменных конечна [42]. Из этой теоремы о линейной вариации индукцией легко получается, что $(n+k-1)$ раз непрерьвно дифференцируемая функция от $n$ переменных имеет конечную $k$-ю вариацию $(1 \leqslant k \leqslant n)$.

$\mathrm{K}$ сожалению, нет формул для вычисления вариаций функций, и потому доказательство этой теоремы оказалось весьма сложным. Трудность не в том, что указывается точная граница гладкости функции, гарантирующей конечность вариации (указанная граница действительно точна). Доказательство не станет проще, если даже предполагать аналитичность функции.

Эта теорема стоила мне двух студенческих лет. Один год - случай $n=3$, второй год $-n=4$, и только при $n=5$ доказательство стало пригодным для общего случая. Оно так и осталось технически сложным, но его идея существенно упростилась. Дело в том, что над малой окрестностью произвольной точки линейная вариация функции соизмерима с линейной вариацией тейлоровского разложения функции в данной точке. Оценка вариации тейлоровского многочлена получается просто, поскольку число компонент его уровня ограничивается константой, определяемой степенью многочлена и числом переменных. Для доказательства было достаточно получить любую сколь угодно грубую оценку числа компонент уровня многочлена. Это не сложно. Однако трудиться по этому поводу не пришлось, поскольку в это время появились упомянутые вьше работы И.Г. Петровского и О.А. Олейник, содержашие точную оценку числа компонент алгебраической поверхности.

9. Оценки $\varepsilon$-энтропии компакта гладких функций. Фиксируем натуральные числа $n$ и $s$ и обозначим через $F$ компакт функций (в равномерной метрике), определенных на $n$-мерном кубе, которые вместе с их частными производными всех порядков до $s$ включительно ограничены по модулю единицей, а через $H_{\varepsilon}-\varepsilon$-энтропию компакта $F$ (логарифм по основанию 2 от числа элементов минимальной $\varepsilon$-сети этого компакта).

Колмогоров доказал (1955 г., [47]) неравенство $A(1 / \varepsilon)^{n / s}<H_{\varepsilon}(F)<B(1 / \varepsilon)^{n / s}$, где $A>0$ и $B>0$ не зависят от $\varepsilon$. 
Теорема о суперпозициях гладких функций (см. раздел 5) может быть легко доказана с помощью этого неравенства. Однако теорема появилась раньше, чем неравенство, и, более того, она стимулировала появление этого неравенства.

Колмогоров доказьвает неравенство, используя лишш локальную формулу Тейлора, опустив при этом оценки вариаций компактов и, соответственно, оценки чисел Бетти. Полученное таким способом новое доказательство теоремы о суперпозициях гладких функций стало более понятньм. И что особенно важно, оценки энтропии оказались полезньми в других аналогичных случаях.

Вычисление энтропии стало модой, и это инициировало разнообразные исследования в теории приближений, теории информации, оценках сложности алгоритмов и т. п. [48]-[79]. Что же касается оценок вариаций множеств, то они оказались весьма полезными в оценках сложности алгоритмов.

10. Пример оценки сложности алгоритмов. Работы по суперпозищиям послужили поводом для ряда исследований по оценке сложности алгоритмов. Для основных классов гладких и аналитических функций тем же методом, что и в случае суперпозищий, вычисляя вариации соответствующих компактов функщий, были получены оценки сложности алгоритмов вычисления функций этих классов (1957 г., [80]-[83]). А именно, при некоторых ограничениях на компакт функций $F$ доказано следуюшее: если алгоритм дает аппроксимацию произвольной функции из $F$ с точностью $\varepsilon$ и при этом число параметров, определяющих функцию, равно $p$ и они входят в формулу для вычисления функции в степени (по совокупности всех параметров) не вьше, чем $k$, то вьполняется неравенство $p \log _{2}(k+1) \geqslant c H_{\varepsilon}$. Здесь $c>0$ - константа, определяемая компактом $F$, а $H_{\varepsilon}-\varepsilon$-энтропия компакта $F$.

Известные схемы аппроксимации гладких и аналитических функций с точки зрения такого рода оценок сложности алгоритмов близки к оптимальньм. Это можно понимать так, что значительно лучших схем аппроксимаций функций этих классов не существует. Приведенные неравенства показывают также, что если при составлении таблицы функции пытаться уменьшить число параметров, определяющих функцию (например, за счет представления функции суперпозищией функций меньшего числа переменных), то это приведет к увеличению степени зависимости от параметров и тем самым к резкому увеличению числа вьполняемых арифметических операций.

Прочитав рукописньй вариант этой работы, Колмогоров прислал мне письмо, в котором поздравил с хорошим результатом, сделав замечание, как подкорректировать формулировку результата. Письмо принесла мне его секретарша (я жил тогда в студенческом общежитии главного здания университета). Такой способ связи меня удивил, поскольку в это время Колмогоров был у себя в кабинете (в центральной части того же здания), и он мог попросить меня прийти к нему. Тем не менее, он прислал письмо, и я воспринял это как его особую оценку полученного результата. А вот с докладом этой работы в Математическом обществе получилось хуже. Колмогоров слишком рано поставил мой доклад, материал был еще сырой. Я плохо подготовил доклад, меня часто перебивали и шумели. Кончилось это тем, что я превратился в зрителя, а Колмогоров, стоя у доски, объяснял содержание работы.

Колмогоров всегда тщательно готовился ко всем своим выступлениям и требовал того же от других. После доклада, получив от него заслуженную взбучку, я попробовал отшутиться: зато на докладе было весело. За это самое “зато” Колмогоров со 
мной с полгода не разговаривал, и я был прощен, лишь когда пришел к нему с очередной работой.

Научные доклады Колмогорова всегда собирали много слушателей, посешаемость его лекций для студентов была тоже хорошей. Однако понимались его лекции с трудом. Он явно переоценивал возможности слушателей. Кстати, он был “удобным" экзаменатором. Он не любил терять время, ожидая ответ на поставленный вопрос, и иногда отвечал сам. Если студент умел удачно обсудить детали этого ответа, то получал хорошую оценку.

11. Кодирование сигналов с конечным спектром. В 1933 году намечали созвать Первьй Всесоюзньй съезд по вопросам реконструкции связи и развития слаботочной промышленности. В. А. Котельников подготовил доклад "О пропускной способности 'эфира' и проволоки в электросвязи”. Съезд не состоялся, но труды были опубликованы [84].

Основной тезис доклада Котельникова состоял в том, что количество информачии, получаемой по каналу связи, пропорчионально ширине полосы пропускаемых частот. Более строгая формулировка этого утверждения назьвается теперь в учебниках теоремой Котельникова: целая функция типа $\sigma$, суммируемая с квадратом на вещественной оси, представима в виде

$$
f(t)=\sum_{k=-\infty}^{\infty} f\left(t_{k}\right) q_{k}(t), \quad \text { где } t_{k}=\frac{k \pi}{\sigma}, \quad q_{k}(t)=\frac{\sin \left(\sigma\left(t-t_{k}\right)\right)}{\sigma\left(t-t_{k}\right)} .
$$

Действительно, из формулы видно, что “количество информации”, т.е. количество независимых чисел, которое дает сигнал со спектром $\sigma$ за единицу времени, равно $\frac{\sigma}{\pi}$.

Такие формулы были известны до Котельникова. Его находка - удачный выбор класса функций и понимание возможности приложений.

Со временем тезис Котельникова неоднократно уточнялся. К. Шеннон характеризовал информационное содержание сигнала, выписав асимптотику плотности кода (длины двоичного кода на единицу времени) для случайного процесса с конечным спектром (1948 г., [85]). Колмогоров получил аналогичную асимптотику для бернштейновского класса функций (вешественных и ограниченных на вешественной оси целых функций конечного типа) (1957 г., [86]). Имеются также оценки плотности кода для сигнала с конечным спектром при некотором специальном выборе метрики, приспособленной для описания высококачественной звукозаписьвающей аппаратуры (1974 г., [87]).

В совместной работе А. Н. Колмогорова и В. М. Тихомирова (1959 г., [58]) доказано, что $\varepsilon$-энтропия (в равномерной метрике) компакта функций, получаемых ограничением на отрезок $[-T, T]$ целых функций типа $\sigma$, вешественных и ограниченных по модулю единицей на вешественной оси, асимптотически равна (при малых значениях $\varepsilon$ и больших значениях $T$ ) величине $\frac{2 T \sigma}{\pi} \log _{2} \frac{1}{\varepsilon}$. Получается так, что если всякая функция указанного класса, передаваемая по данному каналу связи, может быть восстановлена на выходе канала с точностью $\varepsilon$, то количество информации, т.е. число двоичных знаков, которое может быть передано по этому каналу связи за единицу времени, асимптотически равно $\frac{\sigma}{\pi} \log _{2} \frac{1}{\varepsilon}$.

К. Шеннон рано отошел от научной работы, оставаясь, тем не менее, профессором Массачусетского технологического института. Будучи с визитом в Московском университете, Шеннон попросил И. Г. Петровского, который был тогда ректором университета, организовать ему встречу с Колмогоровьм. Встреча состоялась в ректорате, 
в кабинете Петровского. Колмогоров явился в ректорат не в лучшем расположении духа, и беседа оказалась совсем краткой. Возможно, что причиной этого был также и язьковой барьер. Колмогоров владел немецким и французским язьками. Свободно читал на английском, но его разговорный английский был не вполне хорош. Шеннон с ноткой сочувствия высказал сожаление, что они плохо понимают друг друга. Колмогоров ответил, что международных языков пять, он владеет тремя, и если бы его собеседник тоже владел тремя, то проблем бы не было.

12. Цифровая звукозапись. Работы по кодированию сигналов с конечным спектром в полной мере оценили лишь в конце 70-х, когда появилась возможность заменить аналоговую систему записи сигналов цифровой. В частности, в звукозаписи стоимость нужной для этого цифровой аппаратуры стала приемлемой, а качество воспроизведения, получаемое с компакт-дисков, оказалось почти идеальньм. Но всегда остается проблема минимизации сложности алгоритмов. Так, например, в настояшее время в Интернете поток звуковых и видеозаписей стал столь широк, что часто ради экономии объема памяти и времени использования линии связи приходится ограничиваться скромным качеством воспроизведения. Поэтому периодически появляются новые схемы кодирования, уменьшающие объем файлов.

Хорошо иметь возможность слушать музьку не только в концертных залах, но и дома. Сушественным ограничением качества воспроизведения является изменение частотной характеристики воспроизводящей системы, которое получается из-за плохой акустики комнаты. Формула Котельникова при наличии приличного компьютера предоставляет возможность выровнять характеристику. Добиться этого можно, заменив получаемую с проигрьвателя функцию $f(t)$ другой функцией $f^{*}(t)=\sum_{n=-N}^{N} \varepsilon_{n} f\left(t-t_{n}\right)$. Легко видеть, что спектр функции $f^{*}(t)$ (преобразование Фурье этой функции) является произведением двух множителей - спектра функции $f(t)$ и корректирующего множителя, имеющего вид отрезка ряда Фурье, коэффициентами которого являются числа $\varepsilon_{n}$. Поэтому при достаточно большом значении числа $N$ числа $\varepsilon_{n}$ можно выбрать так, чтобы частотная характеристика всей системы, включая акустику комнаты, стала достаточно близкой к константе. Важно то, что не требуется какая-либо предварительная обработка функции $f(t)$. При достаточном быстродействии компьютера поступающая на его вход цепочка чисел $f\left(t_{k}\right)$ в реальном времени будет перерабатьваться в цепочку $f^{*}\left(t_{k}\right)$.

В звукозаписи качество аппаратуры определяется тремя параметрами - шириной полосы воспроизводимых частот, величиной нелинейного искажения сигнала (она измеряется в процентах от средней квадратичной нормы сигнала) и динамическим диапазоном прибора (этот параметр определяется отношением норм максимального и минимального сигналов, для которых гарантируются малые нелинейные искажения). Значения двух последних параметров можно улучшать за счет хорошей точности приближения функции. Но если при этом использовать теорему Котельникова, принимая в качестве кода функции числа $\left\{f\left(t_{k}\right)\right\}$, то длина кода будет возрастать. Получается лучше, если воспользоваться формулой Вейерштрасса, восстанавливающей функцию по ее нулям, приняв в качестве кода координаты нулей.

Оказалось, и этого не ожидали, что существует система кодирования, при которой, не увеличивая длины кода, можно получить сколь угодно широкий динамический диапазон канала связи или прибора. Это обстоятельство дает возможность понять механизм действия имеюшихся систем подавления шума, используемых как 
в аналоговых, так и в цифровых системах записи звука. Эта работа вьполнена мною совместно с В.И. Буслаевым (1974 г., [78]).

Для видеосигналов аналогичных оценок длины кода в зависимости от динамического диапазона канала связи получить пока не удается. Такого рода результат дал бы оценку того, сколь близки к оптимальному методы кодирования, используемые в видеоапаратуре.

\section{СПИСОК ЛИТЕРАТУРЫ}

[1] К.В. Ким, Ю.Е. Нестеров, Б. В. Черкасский. О трудоемкости вычисления градиента // Докл. АН СССР. 1984. Т. 275. № 6. С. 1306-1309.

[2] D. Hilbert. Mathematische Probleme // Nachr. Akad. Wiss. Göttingen. 1900. P. 253-297; // Gesammelte Abhandlungen. V. 3. Berlin: Springer, 1935. P. 290-329.

[3] D. Hilbert. Über die Gleichung neunten Grades // Math. Ann. 1927. V. 97. P. 243-250; // Gesammelte Abhandlungen. V. 2. Berlin: Springer, 1933. P. 393-400.

[4] A. Wiman. Über die Anwendung der Tschirnhausen-Transformation auf die Reduktion algebraischer Gleichungen // Nova Acta Soc. Sci. Upsal. 1928. P. 3-8.

[5] Н. Г. Чеботарёв. Некоторыевопросы в связи с проблемой резольвент // Сборник статей по проблемам резолвент. Т. 1, 1949. С. 255-340.

[6] Колмогоров. Юбилейное издание. Кн. 2. "Этих строк бегущих тесьма ... ". Избранные места из переписки А. Н. Колмогорова и П. С. Александрова / ред.-сост. А. Н. Ширяев. М.: Физматлит, 2003.

[7] А. Н. Колмогоров. О представлении непрерывных функций несколшких переменных суперпозициями непрерьвных функций одного переменного и сложения // Докл. АН СССР. 1957. T. 114. № 5. С. 953-956.

[8] А. Г. Витушкин. Доказательство существования аналитических функций многих переменных, не представимых линейными суперпозициями непрерьвно диффференцируемых функций меньшего числа переменных // Докл. АН СССР. 1964. Т. 156. № 6. С. 1258-1261.

[9] Н. Г. Чеботарёв. О проблеме резольвент // Учен. зап. Казан. гос. ун-та. 1954. Т. 114. C. $189-193$.

[10] В. В. Морозов. Некоторые вопросы в связи с проблемой резольвент // Учен. зап. Казан. гос. ун-та. 1954. Т. 114 . С. $173-187$.

[11] А. Г. Хованский. О суперпозициях голоморфных функций с радикалами // УМН. 1971. T. 26. № 3. C. 213-214.

[12] А. Г. Хованский. О представимости функций в квадратурах // УМН. 1971. Т. 26. № 4. C. $251-252$.

[13] L. Bieberbach. Bemerkung zum dreizehnten Hilbertschen Problem // J. Reine Angew. Math. 1931. V. 165. P. 89-92.

[14] A. Ostrowski. Über Dirichletsche Reihen und algebraische Differentialgleichungen // Math. Z. 1920. V. 8. P. 241-298.

[15] А. Г. Витушкин. Полвека-как один день // УМН. 2002. Т. 57. №1. С. 191-206.

[16] А. Г. Витушкин. К тринадцатой проблеме Гильберта // Докл. АН СССР. 1954. Т. 96. № 4. C. 701-704.

[17] А. Г. Витушкин. Некоторые оценки для вариаций множеств // Докл. АН СССР. 1954. T. 95. № 3. C. 433-434.

[18] A. G. Vitushkin. On representation of functions by means of superpositions and related topics // Enseign. Math. (2). 1977. V. 23. № 3-4. P. 255-320.

[19] А. Н. Колмогоров. О представлении непрерывных функций нескольких переменных суперпозициями непрерьвных функций меньшего числа переменных // Докл. АН СССР. 1956. T. 108. № 2. C. 179-182.

[20] А. С. Кронрод. О функциях двух переменных // УМН. 1950. Т. 5. №1. С. 24-134.

[21] В.И. Арнольд. О функциях трех переменных // Докл. АН СССР. 1957. Т. 114. № 4. C. $679-681$.

[22] В.И. Арнольд. О представлении функций нескольких переменных в виде суперпозиции функций меньшего числа переменных // Матем. просвещение. 1958. № 3. С. 41-61. 
[23] В. И. Арнольд. О представлении непрерьвных функций трех переменных суперпозициями функций двух переменных // Матем. сб. 1959. Т. 48. № 1. С. 3-74.

[24] Ю.П. Офман. О наилучшем приближении функций двух переменных функциями вида $\varphi(x)+\psi(y) / /$ Изв. АН СССР. Сер. матем. 1961. Т. 25. № 2. С. 239-252.

[25] В. П. Моторный. К вопросу о наилучшем приближении функций двух переменных функциями вида $\varphi(x)+\psi(y) / /$ Изв. АН СССР. Сер. матем. 1963. Т. 27. №6. С. 1211-1214.

[26] Г. М. Хенкин. О вложении пространства $s$-гладких функций $n$ переменных в пространство достаточно гладких функций меньшего числа переменных // Докл. АН СССР. 1963. Т. 153. № 1. C. $57-60$.

[27] R. Doss. On the representation of the continuous functions of two variables by means of addition and continuous functions of one variable // Colloq. Math. 1963. V. 10. P. 249-259.

[28] А. Г. Витушкин. Некоторые свойства линейных суперпозиций гладких функций // Докл. AH CCCP. 1964. T. 156. № 5. C. 1003-1006.

[29] Г. М. Хенкин. О линейных суперпозициях непрерывно диффференцируемых функций // Докл. АН СССР. 1964. Т. 157. № 2. С. 288-290.

[30] D. A. Sprecher. On the structure of continuous functions of several variables // Trans. Amer. Math. Soc. 1965. V. 115. P. 340-355.

[31] D. A. Sprecher. On the structure of representation of continuous functions of several variables as finite sums of continuous functions of one variable // Proc. Amer. Math. Soc. 1966. V. 17. P. $98-105$.

[32] А. Г. Витушкин. О возможности представления функций суперпозициями функций меньшего числа переменных // Труды международного конгресса математиков (Москва, 1966). М.: Наука, 1968. С. 322-329.

[33] Л.А.Бассалыго. О представлении непрерывных функций двух переменных при помощи непрерьвных функций одного переменного // Вестник МГУ. Сер. 1. Матем., мех. 1966. № 21. C. $58-63$.

[34] Б. Л. Фридман. Улучшение гладкости функции в теореме А. Н. Колмогорова о суперпозициях // Докл. АН СССР. 1967. Т. 177. № 5. С. 1019-1022.

[35] А. Г. Витушкин, Г. М. Хенкин. Линейные суперпозиции функций // УМН. 1967. T. 22. № 1. C. $77-124$

[36] А. Г. Витушкин. К тринадцатой проблеме Гильберта // Проблемы Гилберта. М.: Наука, 1969. С. 163-170.

[37] Б. Л. Фридман. Нигде не плотность пространства линейных суперпозиций функций нескольких переменных // Изв. АН СССР. Сер. матем. 1972. Т. 36. № 4. С. 814-846.

[38] J.-P. Kahane. Sur le théorème de superposition de Kolmogorov // J. Approx. Theory. 1975. V. 13. № 3. P. 229-234.

[39] G. G. Lorentz. The 13th problem of Hilbert // Proc. Sympos. Pure Math. 1976. V. 28. P. 419-430.

[40] В. Лин. Суперпозиции алгебраических функций // Функц. анализ и его прил. 1976. Т. 10. № 1 . C. $37-45$.

[41] А. А. Милютин. Изоморфность пространств непрерьвных функций над компактами континуальной мощности // Теория функций, функц. анализ и их прил. 1966. № 2. С. 150-156.

[42] А. Г. Витушкин. Вариации функций многих переменных и достаточные условия для их ограниченности // Докл. АН СССР. 1954. Т. 96. №6. С. 1089-1091.

[43] А. Г. Витушкин. О многомерных вариациях. М.: ГИТТЛ, 1955.

[44] Л. Д. Иванов. Вариации множеств и функций. М.: Наука, 1975.

[45] И.Г. Петровский, О.А.Олейник. О топологии действительных алгебраических поверхностей // Изв. АН СССР. Сер. матем. 1949. Т. 13. № 5. С. 389-402.

[46] О. А. Олейник. Оценки чисел Бетти действительных алгебраических гиперповерхностей // Матем. сб. 1952. Т. 28. № 3. С. 635-640.

[47] А. Н. Колмогоров. Оценки минималного числа элементов $\varepsilon$-сетей в различных функциональных классах и их применение к вопросу о представимости функций нескольких переменных суперпозициями функций меньшего числа переменных // УМН. 1955. Т. 10. № 1. C. $192-194$.

[48] А. Н. Колмогоров. О некоторых асимптотических характеристиках вполне ограниченных метрических пространств // Докл. АН СССР. 1956. Т. 108. № 3. С. 385-388. 
[49] А. Н. Колмогоров, А. М. Яглом, И. М. Гельфанд. Количество информации и энтропия для непрерывных распределений // Труды 3-го Всесоюзного математического съезда (Москва, 1956). Т. III: Обзорные доклады. М.: Изд-во АН СССР, 1958. С. 300-320.

[50] А. Г. Витушкин. Абсолютная $\varepsilon$-энтропия метрических пространств // Докл. АН СССР. 1957. Т. 117. № 2. С. 145-148.

[51] В. М. Тихомиров. Об $\varepsilon$-энтропии некоторых классов аналитических функций // Докл. AH CCCP. 1957. T. 117. № 2. C. 191-194.

[52] A. Pełczyński. On the approximation of $S$-spaces by finite dimentional spaces // Bull. Acad. Polon. Sci. Cl. III. 1957. V. 5. №9. P. 879-881.

[53] А. Н. Колмогоров. О линейной размерности топологических векторных пространств // Докл. АН СССР. 1958. Т. 120. № 2. С. 239-242.

[54] К.И. Бабенко. Об энтропии одного класса аналитических функций // Научн. докл. высшей школы. Сер. физ.-матем. наук. 1958. № 2. С. 9-16.

[55] В. Д. Ерохин. О конформных преобразованиях колец и об основном базисе пространства функций, аналитических в элементарной окрестности произвольного континуума // Докл. AH CCCP. 1958. T. 120. № 4. C. 689-692.

[56] В. Д. Ерохин. Об асимптотике $\varepsilon$-энтропии аналитических функций // Докл. АН СССР. 1958. Т. 120. № 5. С. $949-952$.

[57] В. Д. Ерохин. О наилучшем приближении аналитических функций посредством рациональных дробей со свободными полюсами // Докл. АН СССР. 1959. Т. 128. № 1. С. 29-32.

[58] А. Н. Колмогоров, В. М. Тихомиров. $\varepsilon$-энтропия и $\varepsilon$-емкость множеств в функциональных пространствах // УМН. 1959. Т. 14. № 2. С. 3-86.

[59] Ю.А. Брудный, А.Ф. Тиман. Конструктивные характеристики компактных множеств в пространства Банаха и $\varepsilon$-энтропия // Докл. АН СССР. 1959. Т. 126. № 5. C. $927-930$.

[60] Н. С. Бахвалов. О приближенном вычислении кратных интегралов // Вестник МГУ. 1959. № 4. C. 3-18.

[61] В. М. Тихомиров. Поперечники множеств в функциональных пространствах и теория наилучших приближений // УМН. 1960. Т. 15. № 3. С. 81-120.

[62] С. А. Смоляк. $\varepsilon$-энтропия классов $E_{s}^{\alpha, k}(B)$ и $W_{s}^{\alpha}(B)$ в метрике $L_{2} / /$ Докл. АН СССР. 1960. T. 131. № 1. C. 30-33.

[63] Б. С. Митягин. Связь между $\varepsilon$-энтропией, скоростью аппроксимации и ядерностью компакта в линейном пространстве // Докл. АН СССР. 1960. Т. 134. № 4. С. 765-768.

[64] В.Я. Пан. Об аппроксимации аналитических функций рациональными // УМН. 1961. Т. 16. № 5. C. 159-197.

[65] Б.С. Митягин. Аппроксимативная размерность и базисы в ядерных пространствах // УМH. 1961. T. 16. № 4. C. 63-132.

[66] C. Bessaga, A. Pełczyńcki, S. Rolewicz. Approximative dimension of linear topological spaces and some of its applications. (Summary of a report.) // Studia Math. (Ser. Specjalna) Zeszyt. 1963. V. 1. P. 27-29.

[67] Н. С. Бахвалов. Об оценке количества вычислительной работы, необходимой при приближенном решении задач // С. К. Годунов, В. С. Рябенький. Введение в теорию разностных схем. М.: Физматгиз, 1962. С. 316-329.

[68] Н. С. Бахвалов. О скорости сходимости недетерминированных квадратурных процессов на классах функций $W_{p}^{(l)} / /$ Теория вероятн. и ее примен. 1962. Т. 7. № 2. С. 238.

[69] G. G. Lorentz. Lower bounds for the degree of approximation // Trans. Amer. Math. Soc. 1960. V. 97. № 1. P. 25-34.

[70] G. G. Lorentz. Metric entropy, widths, and superpositions of functions // Amer. Math. Monthly. 1962. V. 69. P. 469-485.

[71] В. М. Тихомиров. Работы А. Н. Колмогорова по $\varepsilon$-энтропии функциональных классов и суперпозициям функций // УМН. 1963. Т. 18. № 5. С. 55-92.

[72] Н. М. Коробов. Теоретико-числовые методы в приближенном анализе. М.: Физматгиз, 1963.

[73] С. А. Смоляк. Квадратурные интерполяционные формулы на тензорных произведениях некоторых классов функций // Докл. АН СССР. 1963. Т. 148. № 5. С. 1042-1045. 
[74] Ю. А. Брудный, Б. Д. Котляр. О порядке роста $\varepsilon$-энтропии на некоторых компактных классах функций // Докл. АН СССР. 1963. Т. 148. № 5. С. 1001-1004.

[75] Ю. П. Офман. О приближенной реализации непрерывных функций на автоматах // Докл. АН СССР. 1963. Т. 152. № 4. С. 823-826.

[76] H.S. Shapiro. Some negative theorems of approximation theory // Michigan Math. J. 1964. V. 11. № 3. P. 211-217.

[77] P. A. Ostrand. Dimension of metric Spaces and Hilbert's problem 13 // Bull. Amer. Math. Soc. 1965. V. 71. № 4. P. 619-622.

[78] А. Г. Витушкин, В.И. Буслаев. Оценка длины кода сигналов с конечным спектром в связи с задачами звукозаписи // Изв. АН СССР. Сер. матем. 1974. Т. 38. № 4. С. 867-895.

[79] В.В.Змушко. Энтропия класса целшх функций с частотно-зависимой метрикой // Изв. АН СССР. Сер. матем. 1976. Т. 40. № 5. С. 1173-1186.

[80] А. Г. Витушкин. Некоторые оценки из теории табулирования // УМН. 1957. Т. 12. № 2. C. $227-228$.

[81] А. Г. Витушкин. Некоторые оценки из теории табулирования // Докл. АН СССР. 1957. T. 114. № 5. C. $923-926$.

[82] А.Г. Витушкин. О наилучших приближениях дифференцируемых и аналитических функций // Докл. АН СССР. 1958. Т. 119. № 3. С. 418-420.

[83] А. Г. Витушкин. Оценка сложности задачи табулирования. М.: Физматгиз, 1959.

[84] В. А. Котельников. О пропускной способности "эфора" и проволоки в электросвязи // Материалы к 1-му Всесоюзному съезду по вопросам реконструкции дела связи и развития слаботочной промышленности. М.: Управление связи РКKА, 1933.

[85] C. E. Shannon. A mathematical theory of communication // Bell. System Tech. J. 1948. V. 27. P. 379-423; 623-656.

[86] А.Н. Колмогоров. Теория передачи информации // Сессия АН СССР по научным проблемам автоматизации производства. Т. 1: Пленарные заседания. М.: Изд-во АН СССР, 1957. C. $66-99$.

[87] A. G. Vitushkin. Coding of signals with finite spectrum and sound recording problems // Proceedings of the International Congress of Mathematicians (Vancouver, 1974). V. 1. Montréal: Canad. Math. Congress, 1975. P. 221-226. 